\title{
Simultaneous Combination Treatment Using High-Intensity Focused Ultrasound and Fractional Carbon Dioxide Laser Resurfacing for Facial Rejuvenation
}

\author{
Hee Yong Kang \\ Eun Soo Park \\ Seung Min Nam
}

Department of Plastic \& Reconstructive Surgery, Soonchunhyang University Bucheon Hospital, Bucheon, Korea
Received June 24, 2019

Accepted June 26, 2019

\footnotetext{
Correspondence

Eun Soo Park

Department of Plastic and Reconstructive Surgery, Soonchunhyang University Bucheon Hospital, 170 Jomaru-ro, Bucheon 14584, Korea Tel.: +82-32-621-5319

Fax: +82-32-621-5016

E-mail: peunsoodaschmc.ac.kr

(C) Korean Society for Laser Medicine and Surgery

(c) This is an open access article distributed under the terms of the Creative Commons Attribution NonCommercial License (http://creativecommons.org/ licenses/by-nc/4.0) which permits unrestricted noncommercial use, distribution, and reproduction in any medium, provided the original work is properly cited.
}

\begin{abstract}
Background and Objectives
High-intensity focused ultrasound (HIFU) can produce small zones of thermal damage. A HIFU procedure is non-invasive and it can achieve rejuvenation of facial skin. Fractional $\mathrm{CO}_{2}$ laser resurfacing delivers thermal damage to the pixilated columnar zone of the skin and so evoke collagen remodeling, the same as HIFU. In many cases, the patients who want rejuvenation with HIFU are also good candidates for cutaneous photorejuvenation such as can be accomplished via fractional $\mathrm{CO}_{2}$ resurfacing. If patients are treated in a single session by remodeling both the superficial and deep compartments of skin by using both modalities, then improvement in rhytides and tightening of sagging skin will optimize the aesthetic result.

\section{Materials and Methods \\ Between May 2014 and January 2018, a total of 44 patients were treated with combination $\mathrm{HIFU}$ and fractional $\mathrm{CO}_{2}$ laser resurfacing according to our protocol. First, the HIFU was applied to the entire face with an average of 300 treatment lines. Immediately after HIFU treatment, the ultrasound gel was washed off and then fractional $\mathrm{CO}_{2}$ laser resurfacing was performed. We evaluated the patients using 4-point grading scales. The clinician examined the skin for evidence of complications after the completion of treatment.}

\section{Results}

All the patients' skin quality showed improvement. Further. the clinical results after duel modality treatment were substantially better than that after the use of either modality alone. The recovery times and the incidence of adverse events when quickly and consecutively performing both treatments were similar as compared to those with employing stepwise treatment. We encountered no complications whatsoever.

\section{Conclusion}

When compared with stepwise therapy, combination therapy with HIFU and fractional $\mathrm{CO}_{2}$ resurfacing offers better, safer and more effective clinical results. Thus, for targeting multiple layers of aging facial skin, this combination therapy can be safely performed in a single treatment session.

\section{Key words}

Rejuvenation; Fractional $\mathrm{CO}_{2}$ Laser; High-Intensity Focused Ultrasound Ablation; Asian Continental Ancestry Group; Cosmetic Techniques 


\section{INTRODUCTION}

Recently in older people, there has been increasing public concern about aging skin. The signs of aging skin are dermal collagen and elastin fragmentation, fibroblast dysregulation, abnormal production of collagen-degrading matrix metalloproteinases and decreased assembly of Type I collagen, appeared as skin laxity, rhytides, and fine lines. ${ }^{1,2}$

High-intensity focused ultrasound (HIFU) has been researched as a tool for the treatment of solid tumor, originally. ${ }^{3}$ In recent years, HIFU was developed as effective and noninvasive skin rejuvenation treatment device. HIFU produces thermal injury at precise depths in the deep dermis to the SMAS layer while sparing superficial skin layer. This procedure can improve skin laxity and texture and have been preferred by patients who avoid invasive or surgical skin tightening.

Ablative fractional laser resurfacing with $\mathrm{CO}_{2}$ source represents preferred procedure in the treatment of aging facial skin. This technology delivers thermal injury into the epidermis and papillary dermis, causing wound healing process. Collagen remodeling and neo-collagenesis occurs at a more superficial level than HIFU and wrinkles and fine lines improve.

In author's experiences, most of patients who received HIFU treatment are also good indication of cutaneous rejuvenation such as fractional $\mathrm{CO}_{2}$ resurfacing. So author think if we treat with HIFU and fractional $\mathrm{CO}_{2}$ laser at the same time, entire skin layer to SMAS are remodeled simultaneously and occurred more improvement of aesthetic results than single modality treatment. Many studies about single modality's effectiveness have been reported, but study about combination treatment of these are rare, especially in Asian ethnics.

Herein, we report about therapeutic efficacy and safety of HIFU and fractional $\mathrm{CO}_{2}$ laser combination treatment to large number of aging Asian patients.

\section{MATERIALS AND METHODS}

\section{Treatment procedure}

This study was performed by retrospective review. Between May 2014 and January 2018, total 44 Korea patients were treated at Department of Plastic and Reconstructive Surgery of Soonchunhyang University Bucheon Hospital. Patient's Fitzpatrick skin types are III to V with skin laxity and wrinkles. Patients with history of other cosmetic procedure were excluded from this study. We use the HIFU (Ultraformer $^{\circledR}$, Classys Inc., Seoul, Korea) and fractional
$\mathrm{CO}_{2}$ laser (Line-Xel ${ }^{\mathrm{TM}}$, United thech Inc.).

Analgesia protocols was performed with topical application of $5 \%$ lidocaine cream (EMLA, AstraZeneca, Sdertlje, Sweden) 60 minutes before treatment. Then immediately before the procedure, ointment was washed off with water and soap.

Next, a thin layer of ultrasound gel was applied to face. Operator situated HIFU transducer perpendicularly on the target face skin and pressed the transducer uniformly. Authors used two types of transducer. First, treatment was applied by $4-\mathrm{MHz}, 4.5 \mathrm{~mm}$ depth transducers with $0.7-1.0 \mathrm{~J} / \mathrm{mm}^{2}$ to thick skin area like cheek and chin. Then 7-MHz, $3.0 \mathrm{~mm}$ depth transducers with 0.3-0.5 J/ $\mathrm{mm}^{2}$ was used in thick skin area and relatively thin skin area like forehead, nose and periorbital area. Ultrasound pulses being delivered to individual line within 1-2 seconds. Distance between lines was maintained at 3 to 5 $\mathrm{mm}$ parallel position to the previous treatment line. HIFU was applied to the entire face with average 320 treatment lines. The number of HIFU lines delivered necessary for treatment varied with the individual's pain tolerance and the size of the face skin areas. $80 \%$ of total shots were applied to thick skin area; $40 \%$ of shots by $4.5 \mathrm{~mm}$ depth transducer and $40 \%$ of shots by $3 \mathrm{~mm}$ depth transducer. Remnant $20 \%$ of shots by $3 \mathrm{~mm}$ depth transducer were applied to thin skin area. After HIFU treatment, the ultrasound gel was washed off. A fractional $\mathrm{CO}_{2}$ laser was applied immediately to the face with 100-120 mJ fluence. The laser procedure was performed with a pulse duration of 100-180 $\mu$ s with a distance of 0.8-1 mm, degree 1 th. Initial laser was applied in square shape with a size of $25 \times 25 \mathrm{~mm}$. Then $10 \times 10 \mathrm{~mm}$ size square shape laser was applied in random shot mode to remnant face surface. The clinical endpoint of treatment was the presence of slight erythema. Complete treatment time was average 15 minutes. After laser procedure, cool pack compresses and postoperative $4 \%$ hydroquinone and sunscreen were applied to treatment area. Color make-up was permitted after $1-2$ weeks.

\section{Evaluation}

Digital photographs (Nikon D750, Tokyo, Japan) before and after 3 months were collected. For each photo camera settings and identical ambient lighting were maintained in a same photographic room. Treatment effect was evaluated by three blinded independent plastic surgeons. They evaluated paired before and after digital photographs by using the Modified Quantitative Comprehensive Grading Scale (MQCGS) (Table 1). AlexiadesArmenakas et al. extensively used Quantitative Compre- 
Table 1. Modified quantitative comprehensive grading scale

\begin{tabular}{|c|c|c|c|c|c|}
\hline \multirow{2}{*}{$\begin{array}{l}\text { Grading } \\
\text { scale }\end{array}$} & \multirow{2}{*}{$\begin{array}{l}\text { Descriptive } \\
\text { Parameter }\end{array}$} & \multicolumn{4}{|c|}{ Skin aging } \\
\hline & & Rhytides & Laxity & Elastosis & Texture \\
\hline 1 & Mild & Wrinkles in motion : superficial & Localized : NL or ML & Early, Minimal yellow hue & Mild irregularity \\
\hline 2 & Moderate & $\begin{array}{l}\text { Wrinkles at rest : localized, } \\
\text { superficial }\end{array}$ & Localized : NL, ML, Jowls, SM & $\begin{array}{l}\text { Yellow hue, Localized PO and } \\
\text { malar EB }\end{array}$ & $\begin{array}{l}\text { Rough in several localized } \\
\text { areas }\end{array}$ \\
\hline 3 & Advanced & $\begin{array}{l}\text { Wrinkles at rest : multiple } \\
\text { superficial, a few deep }\end{array}$ & Prominent: NL, ML, Jowls, SM & $\begin{array}{l}\text { Yellow hue, multiple EB with little } \\
\text { uninvolved skin }\end{array}$ & $\begin{array}{l}\text { Rough with a few uninvolved } \\
\text { skin }\end{array}$ \\
\hline 4 & Severe & $\begin{array}{l}\text { Wrinkles throughout: multiple } \\
\text { deep }\end{array}$ & Marked : NL, ML, Jowls, SM & Deep yellow hue, extensive EB & Extensively rough \\
\hline
\end{tabular}

EB, elastotic beads; ML, melolabial; NL, nasolabial; PO, periorbital; SM, submental/submandibular.

Table 2. Subject Global Aesthetic Improvement Scale (SGAIS)

\begin{tabular}{cll}
\hline Score & \multicolumn{1}{c}{ Rating } & \multicolumn{1}{c}{ Definition } \\
\hline 1 & Worse & No improvement \\
2 & Mildly improved & $<25 \%$ improvement \\
3 & Improved & $25-49 \%$ improvement \\
4 & Much improved & $50-74 \%$ improvement \\
5 & Very much improved & $75 \%$ improvement or more \\
\hline
\end{tabular}

Table 3. Patients characteristics

\begin{tabular}{lc}
\hline Characteristic & Value \\
\hline Sex (female, male) & 30,14 \\
Mean age (range) & $51.2(38-63)$ \\
Fitzpatrick skin type & Type $3: 23$ \\
& Type $4: 19$ \\
& Type $5: 2$ \\
\hline
\end{tabular}

hensive 4-point Grading Scale for evaluating laser and energy-based cosmetic treatments. ${ }^{4-7}$ Author's modification was done by excluding categories of dyschromia, erythema-telangiectasia and keratosis, and combining integer and decimal scale. The improvement for each patient was calculated as the difference between the mean baseline and mean follow-up grades. Also subjective evaluation was assessed by using the Subject Global Aesthetic Improvement Scale (SGAIS) (Table 2) 3 months after treatment. Also at the same time, all subjects rated their satisfaction as excellent, good, fair or poor. The clinician examined the skin for evidence of edema, erythema, hypopigmentation, and hyperpigmentation after treatment.

\section{Statistical analysis}

Statistical analyses of comparisons before and after the treatment by using Modified Quantitative Comprehensive Grading Scale were performed using the Paired t-test
Mean MQCGS

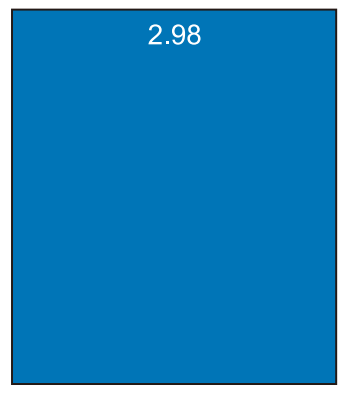

Pre-treatment

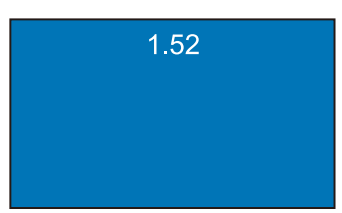

Post-treatment
Fig. 1. Mean value of Modified Quantitative Comprehensive Grading Scale before and after treatment.

and SPSS statistical software (SPSS Inc., version 20.0, Chicago, IL, USA).

\section{RESULTS}

This study included 44 Korean patients ( 30 women and 14 men), aged 38 to 63 years (mean, 51.2 years) and All 44 subjects returned for the 90-day follow-up. Patient's Fitzpatrick skin type are 3-5 type. Dermographics of patient is shown in Table 3. Skin quality was significantly improved after treatment, as demonstrated by the MQCGS, which was evaluated by 3 physicians blinded to the patients. (Fig. 1) The mean MQCGS scores were 2.98 (standard deviation 0.59) before treatment and 1.52 (standard deviation 0.37) 3 months after the treatment. After combination treatment, the mean MQCGS values decreased significantly l $p$ $<0.05$ ). All subjects showed clinical improvement after 3 months. Their average SGAIS was 3.93. Sixteen patients (36\%) were assessed as having very much improvement, fourteen patients (32\%) as having much improvement, nine patients $(21 \%)$ as having fair improvement, and five patients (11\%) as having mildly improvement at the 


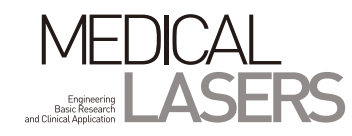

3-month follow up visit. No subject was reported to show no improvement. Post-treatment photographic finding was shown in Fig. 2, 3. Patient's satisfaction results were marked in Fig. 4. The percentage of 'Excellent' were the highest value and there are no patients who answered 'Poor'. Side effects included purpura, edema, erythema and serous discharge lasting 2 to 7 days (average 4 days) in all patients. No adverse effects or complications, such as scarring, infections, hypopigmentation and hyperpigmentation occurred in the present study. Recovery times and the incidence of adverse events was similar when compared with stepwise treatment.
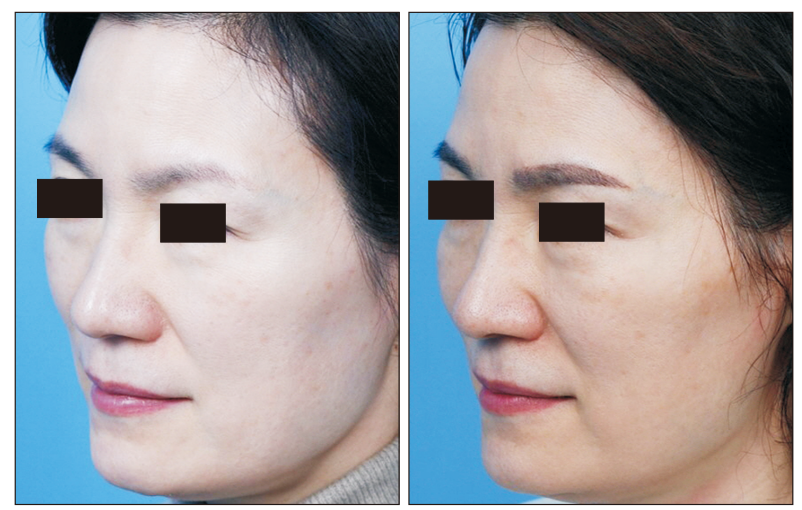

Fig. 2. These are 56 years old female patient's photo. Left is oblique view before treatment. Right photo is oblique view after treatment 3 months. Improvement of periorbital and malar wrinkles and tightening effect of submandibular area skin are shown.

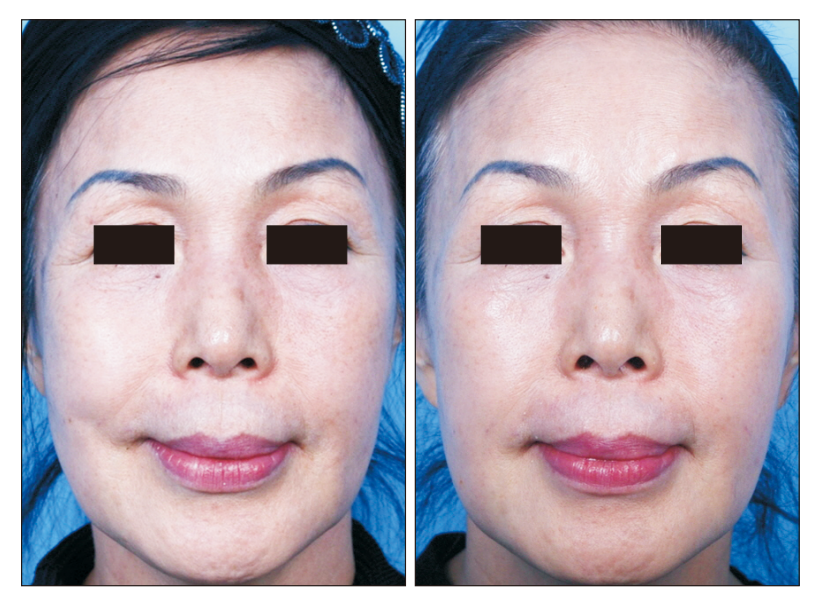

Fig. 3. These are 63 years old female patient's photo. Left is AP view before treatment. Right photo is AP view after treatment 3 months. Improvement of periorbital wrinkles and tightening effect of forehead, nasolabial and submandibular area skin are shown.

\section{DISCUSSION}

As human grows older, constantly aging process progressed in skin and its under structural tissues. The number of fibroblast and collagen synthesis also decreases and various skin appendages are also dropped. These aging process can present clinically as features of sunspots, uneven skin color, wrinkle and sagging skin.

"Rejuvenation" means restoration of youth and making looking young, including the treatment for the improvement of skin color, quality and elasticity of the skin as well as diminishing winkles and tightening the aging skin. The development of medical device for rejuvenation can be classified in many different ways.

In 2004, Manstein et al. introduced a fresh concept of rejuvenation utilizing a laser, now known as fractional photothermolysis. ${ }^{8}$ In 2007, Hantash et al. introduced fractional laser based on fractional photothermolysis theory. Many fractional lasers are now widely used in clinical practice of rejuvenation treatment. Especially, ablative fractional $\mathrm{CO}_{2}$ laser causes vaporization, making areas of tissue coagulation spanning the epidermis and papillary dermis. Shrinkage of the collagen fibers with consequent shrinking of the skin and neo-collagenesis occurs and wrinkles and fine lines improve. ${ }^{10-12}$ Compared to ablative fractional $\mathrm{CO}_{2}$ laser, HIFU is a non-ablative rejuvenation technique. This uses the energy of ultrasound wave, which penetrates the epidermal layer and induces vibration of cellular molecules. These energy heats tissue to greater than $60^{\circ}$, producing small $\left(<1 \mathrm{~mm}^{3}\right.$ ) microthermal lesions at precise depths in the reticular dermis up to the SMAS layer while sparing epidermis and papillary dermis. This process provokes tissue coagulation, col-

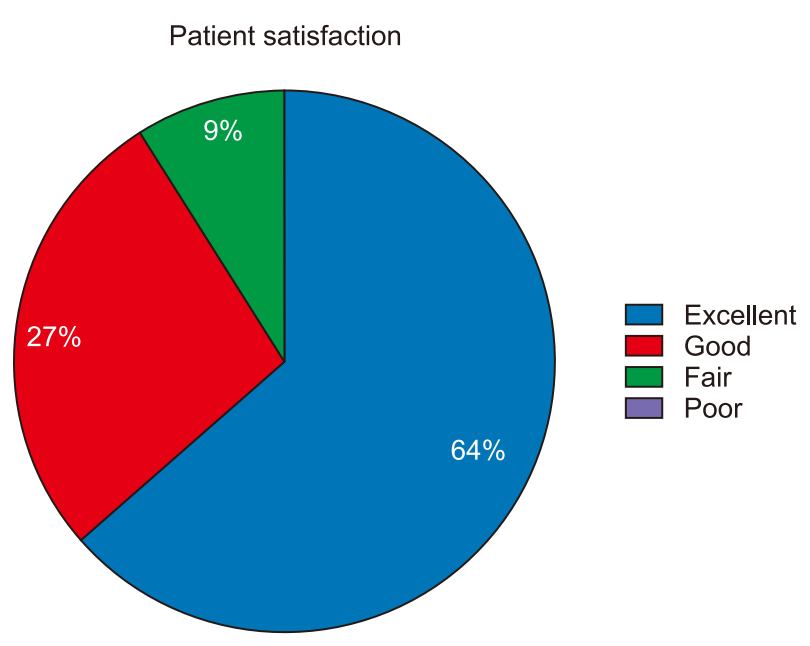

Fig. 4. Patient's satisfaction. 
lagen denaturation and contracture with subsequent neocollagenesis and collagen structure remodeling. ${ }^{13-15}$

Rejuvenation using non-invasive or minimally invasive procedure like $\mathrm{HIFU}$ or fractional $\mathrm{CO}_{2}$ laser is superior to surgical procedure in terms of pain, rapid return to daily life, short recovery time and low risk of adverse events. Because of these advantages, many patients who desire a skin-tightening procedure prefer noninvasive skin tightening to invasive or surgical skin tightening. However, these procedures cannot get dramatic effect in one time, so repetitive treatment at interval several months, or combination of other aesthetic procedures should be forced for ideal appearance. So authors hypothesize that if HIFU and ablative fractional $\mathrm{CO}_{2}$ laser are performed in one time without discomfort and post-treatment complications, patient's aging skin can more dramatically improves and patient's satisfaction will more increase because of timesaving and better aesthetic result.

In 2014, there was the study about combination treatment applying similar concept. ${ }^{16}$ They used microfocused ultrasound device instead of HIFU and different fractional $\mathrm{CO}_{2}$ laser device. They concluded combination treatment is safe and effectiveness procedure to patients. However, there was several limitations of this study, including lack of standardized evaluations and statistical proof. Besides, their patients consist of Caucasians. Asian people's dark skin is thicker and has more compact dermis than Caucasian's white skin, with the thickness being proportional to the degree of pigmentation. ${ }^{17}$ Moreover dark skin types have more cornified cell layers and greater lipid content compared to white skin types. ${ }^{18,19}$ So the experience gained from applying similar treatment protocols in Caucasians has been found not to be totally compatible to Asian skin. Thus, we start study about effect and safety of combination treatment to Asian skin using different devices.

Each modality's rejuvenation effects were different according to target layer. However, in combination treatment multiple layers were addressed at once, theoretically combination treatment's clinical results are more substantive than after the use of either modality alone. So When considering combination treatment, we should concern initially about complication like scarring, because the extensive heat load is applied to the underside and surface of the skin. But in our study data, there is no adverse effect. This shows combination treatment is a safe over the face of Asian, targeting both the deeper fibromuscular layer with HIFU and the more superficial dermis of the skin with fractional $\mathrm{CO}_{2}$ laser.

Our study is the first study of combination treatment of
HIFU and fractional $\mathrm{CO}_{2}$ laser in facial rejuvenation using standardized evaluation methods with statistics. In our study data, standardized scales of skin quality (rhytides, laxity, elastosis and texture) were improvement with statistically significance by one treatment session and most of patient's subjective evaluation was high score. Also, most of patients were satisfied to this procedure, because procedure is safe, short-time and gives more dramatic effect than using each modality. Thus, we recommend our simultaneous combination treatment with HIFU and fractional $\mathrm{CO}_{2}$ laser protocols in Asian face rejuvenation treatment.

Further study about combination treatment with HIFU and fractional $\mathrm{CO}_{2}$ laser will be done for verifying the best treatment protocols, possibility of applying other anatomical site, difference by patient's race and comparing with other energy-based treatment device like radiofrequency, variable wavelength laser, etc.

\section{CONCLUSION}

Simultaneous Combination Treatment with HIFU and fractional $\mathrm{CO}_{2}$ laser on the face is a safe and effective method for targeting multiple layers of facial skin aging and can be safely performed in a single treatment session.

\section{CONFLICT OF INTEREST}

The authors declare that they have no conflicts of interest to disclose.

\section{REFERENCES}

1. Fisher GJ, Varani J, Voorhees JJ. Looking older: fibroblast collapse and therapeutic implications. Arch Dermatol 2008;144:666-72.

2. Quan T, Little E, Quan H, Qin Z, Voorhees JJ, Fisher GJ. Elevated matrix metalloproteinases and collagen fragmentation in photodamaged human skin: impact of altered extracellular matrix microenvironment on dermal fibroblast function. J Invest Dermatol 2013;133:1362-6.

3. Laubach HJ, Makin IR, Barthe PG, Slayton MH, Manstein D. Intense focused ultrasound: evaluation of a new treatment modality for precise microcoagulation within the skin. Dermatol Surg 2008;34:727-34.

4. Alexiades-Armenakas M. Rhytides, laxity, and photoaging treated with a combination of radiofrequency, diode laser, and pulsed light and assessed with a comprehensive grading scale. J Drugs Dermatol 2006;5:731-8. 
5. Alexiades-Armenakas M. Nonablative skin tightening with a variable depth heating 1310-nm wavelength laser in combination with surface cooling. J Drugs Dermatol 2007;6:1096-103.

6. Alexiades-Armenakas M, Dover JS, Arndt KA. Unipolar versus bipolar radiofrequency treatment of rhytides and laxity using a mobile painless delivery method. Lasers Surg Med 2008;40:446-53.

7. Alexiades-Armenakas M. Assessment of the mobile delivery of infrared light (1100-1800 nm) for the treatment of facial and neck skin laxity. J Drugs Dermatol 2009;8:221-6.

8. Manstein D, Herron GS, Sink RK, Tanner H, Anderson RR. Fractional photothermolysis: a new concept for cutaneous remodeling using microscopic patterns of thermal injury. Lasers Surg Med 2004;34:426-38.

9. Hantash BM, Mahmood MB. Fractional photothermolysis: a novel aesthetic laser surgery modality. Dermatol Surg 2007;33:525-34.

10. Prignano F, Bonciani D, Campolmi P, Cannarozzo G, Bonan P, Lotti T. A study of fractional CO@ü laser resurfacing: the best fluences through a clinical, histological, and ultrastructural evaluation. J Cosmet Dermatol 2011;10:210-6.

11. Longo C, Galimberti M, De Pace B, Pellacani G, Bencini PL. Laser skin rejuvenation: epidermal changes and collagen remodeling evaluated by in vivo confocal microscopy. Lasers Med Sci 2013;28:769-76.

12. Berlin AL, Hussain M, Phelps R, Goldberg DJ. A prospective study of fractional scanned nonsequential carbon dioxide laser resurfacing: a clinical and histopathologic evaluation. Dermatol Surg 2009;35:222-8.

13. Alam M, White LE, Martin N, Witherspoon J, Yoo S, West DP. Ultrasound tightening of facial and neck skin: a rater-blinded prospective cohort study. J Am Acad Dermatol 2010;62:262-9.

14. Suh DH, Shin MK, Lee SJ, Rho JH, Lee MH, Kim NI, et al. Intense focused ultrasound tightening in Asian skin: clinical and pathologic results. Dermatol Surg 2011;37:1595-602.

15. Lee HS, Jang WS, Cha YJ, Choi YH, Tak Y, Hwang E, et al. Multiple pass ultrasound tightening of skin laxity of the lower face and neck. Dermatol Surg 2012;38:20-7.

16. Woodward JA, Fabi SG, Alster T, Colón-Acevedo B. Safety and efficacy of combining microfocused ultrasound with fractional CO2 laser resurfacing for lifting and tightening the face and neck. Dermatol Surg 2014;40 Suppl 12:S190-3.

17. Montagna W, Prota G, Kenney JA. The structure of black skin. In: Montagna W, Prota G, Kenney JA, editors. Black skin : structure and function. San Diego: Academic Press; 1993.

18. Sugino K, Imokawa G, Maibach HI. Ethnic difference of stratum corneum lipid in relation to stratum corneum function. J Invest Dermatol 1993;100:587.

19. Berardesca E, de Rigal J, Leveque JL, Maibach HI. In vivo biophysical characterization of skin physiological differences in races. Dermatologica 1991;182:89-93. 